\title{
CORRECTION
}

\section{Correction to: Intensive care unit length of stay is reduced by protocolized family support intervention: a systematic review and meta-analysis}

\author{
Hyun Woo Lee ${ }^{1} \mathbb{B}$, Yeonkyung Park ${ }^{2}$, Eun Jin Jang ${ }^{3}$ and Yeon Joo Lee Le $^{*}$
}

@ 2019 Springer-Verlag GmbH Germany, part of Springer Nature

\section{Correction to: Intensive Care Med (2019) 45:1072-1081 https://doi.org/10.1007/s00134-019-05681-3}

The original version of this article unfortunately contained a mistake. One of the affiliations was incorrect: Division of Pulmonary and Critical Care Medicine, Department of Internal Medicine, Veterans Health Service Medical Center, Portland, USA.

The correct affiliation is: Division of Pulmonary and Critical Care Medicine, Department of Internal Medicine, Veterans Health Service Medical Center, Seoul, South Korea.

The Publisher apologizes for the mistake.
*Correspondence: yjlee1117@snubh.org

${ }^{4}$ Division of Pulmonary and Critical Care Medicine, Department of Internal Medicine, Seoul National University College of Medicine, Seoul National University Bundang Hospital 82, Gumi-ro 173 Beon-gil, Bundang-gu, Seongnam-si 13620, South Korea

Full author information is available at the end of the article

\section{Author details}

${ }^{1}$ Division of Pulmonary and Critical Care Medicine, Department of Internal Medicine, Seoul National University College of Medicine, Seoul National University Hospital, Seoul, South Korea. ${ }^{2}$ Division of Pulmonary and Critical Care Medicine, Department of Internal Medicine, Veterans Health Service Medical Center, Seoul, South Korea. ${ }^{3}$ Department of Information Statistics, Andong National University, Andong, South Korea. ${ }^{4}$ Division of Pulmonary and Critical Care Medicine, Department of Internal Medicine, Seoul National University College of Medicine, Seoul National University Bundang Hospital 82, Gumi-ro 173 Beon-gil, Bundang-gu, Seongnam-si 13620, South Korea.

Published online: 8 October 2019 